\title{
Cross-Modal Attention Effects in the Vestibular Cortex during Attentive Tracking of Moving Objects
}

\author{
Sebastian M. Frank, ${ }^{1}$ Liwei Sun, ${ }^{1}$ Lisa Forster, ${ }^{2}$ Peter U. Tse, ${ }^{1}$ and ${ }^{\circledR}$ Mark W. Greenlee ${ }^{2}$ \\ ${ }^{1}$ Department of Psychological and Brain Sciences, Dartmouth College, Hanover, New Hampshire 03755, and ${ }^{2}$ Institute of Experimental Psychology, \\ University of Regensburg, 93053 Regensburg, Germany
}

The midposterior fundus of the Sylvian fissure in the human brain is central to the cortical processing of vestibular cues. At least two vestibular areas are located at this site: the parietoinsular vestibular cortex (PIVC) and the posterior insular cortex (PIC). It is now well established that activity in sensory systems is subject to cross-modal attention effects. Attending to a stimulus in one sensory modality enhances activity in the corresponding cortical sensory system, but simultaneously suppresses activity in other sensory systems. Here, we wanted to probe whether such cross-modal attention effects also target the vestibular system. To this end, we used a visual multiple-object tracking task. By parametrically varying the number of tracked targets, we could measure the effect of attentional load on the PIVC and the PIC while holding the perceptual load constant. Participants performed the tracking task during functional magnetic resonance imaging. Results show that, compared with passive viewing of object motion, activity during object tracking was suppressed in the PIVC and enhanced in the PIC. Greater attentional load, induced by increasing the number of tracked targets, was associated with a corresponding increase in the suppression of activity in the PIVC. Activity in the anterior part of the PIC decreased with increasing load, whereas load effects were absent in the posterior PIC. Results of a control experiment show that attention-induced suppression in the PIVC is stronger than any suppression evoked by the visual stimulus per se. Overall, our results suggest that attention has a cross-modal modulatory effect on the vestibular cortex during visual object tracking.

Key words: area PIC; area PIVC; attentional tracking; vestibular cognition; vestibular cortex

\section{Significance Statement}

In this study we investigate cross-modal attention effects in the human vestibular cortex. We applied the visual multipleobject tracking task because it is known to evoke attentional load effects on neural activity in visual motion-processing and attention-processing areas. Here we demonstrate a load-dependent effect of attention on the activation in the vestibular cortex, despite constant visual motion stimulation. We find that activity in the parietoinsular vestibular cortex is more strongly suppressed the greater the attentional load on the visual tracking task. These findings suggest cross-modal attentional modulation in the vestibular cortex.

\section{Introduction}

We know far less about the cortical organization of the human vestibular system than we do about other human sensory systems. Increasing evidence points to the region that includes the

Received Aug. 4, 2016; revised 0ct. 26, 2016; accepted 0ct. 29, 2016.

Author contributions: S.M.F., L.S., P.U.T., and M.W.G. designed research; S.M.F. and L.F. performed research; S.M.F. analyzed data; S.M.F., L.S., P.U.T., and M.W.G. wrote the paper.

This work was supported by the Alexander von Humboldt Foundation (to P.U.T. and S.M.F.), by the Deutsche Forschungsgemeinschaft (to M.W.G.), and by a Harris German/Dartmouth distinguished visiting professorship of Dartmouth College (to M.W.G.). We thank Christian Renner from the workshop at the University of Regensburg for constructing and maintaining the caloric stimulation device. We also thank two anonymous reviewers for helpful comments on a previous version of this manuscript.

The authors declare no competing financial interests.

Correspondence should be addressed to Mark W. Greenlee, Institute of Experimental Psychology, University of Regensburg, 93053 Regensburg, Germany. E-mail: mark.greenlee@ur.de.

DOI:10.1523/JNEUROSCI.2480-16.2016

Copyright $\odot 2016$ the authors $\quad 0270-6474 / 16 / 3612720-09 \$ 15.00 / 0$ midposterior fundus of the Sylvian fissure and the posterior insula (Guldin and Grüsser, 1998; Kahane et al., 2003; Lopez and Blanke, 2011; Lopez et al., 2012; zu Eulenburg et al., 2012; Dieterich and Brandt, 2015; Greenlee et al., 2016) as particularly responsive to vestibular stimuli (e.g., caloric or galvanic stimuli). Two separate vestibular areas have been identified in this region: the parietoinsular vestibular cortex (PIVC) and the posterior insular cortex (PIC; for nonhuman primate results, see Chen et al., 2010, 2011; Shinder and Newlands, 2014; for human imaging results, see Frank et al., 2014, 2016).

Previous studies have examined these areas not only with vestibular, but also with visual stimuli, e.g., visual object motion cues. This has revealed an interesting functional difference between the PIVC and PIC: observing an object in motion enhances activity in the PIC (Sunaert et al., 1999; Orban et al., 2003; Frank et al., 2014, 2016), but suppresses activity in the PIVC (Deutschländer et al., 2002; Kleinschmidt et al., 2002; Frank et al., 2016). 
While these imaging studies were informative in demonstrating cross-modal suppression of activity in the PIVC during stimulation with a visual cue (i.e., visual object motion), they did not examine whether this effect can be further augmented by attention. It is well established that attention can have cross-modal effects on activity. For instance, attention to a visual stimulus, even without stimulus presentation, is associated with simultaneous suppression of activity in the auditory cortex (Mozolic et al., 2008). There are indications for a similar cross-modal suppression effect of visual attention in the region where the vestibular cortex is located (Tomasi et al., 2006). However, no explicit investigation of the vestibular cortex, including a separation between areas PIVC and PIC, has been performed to date.

Therefore, in this study, we investigated cross-modal attention effects in the human vestibular cortex with a focus on areas PIVC and PIC. To this end, we used an attentional tracking paradigm with varying numbers of visual target objects to be tracked (Pylyshyn and Storm, 1988; Cavanagh and Alvarez, 2005). In this paradigm, a subset of independently moving stimuli is temporarily cued at the beginning of a trial and participants are asked to track these targets covertly among distractors for a fixed duration. Targets and distractors are physically identical and differ only based on their pretracking designation as target or distractor. At the end of each trial, just one of the moving stimuli is cued and participants indicate whether it was a tracked target or a distractor.

Previous studies have used this paradigm to examine changes in brain activity as a function of attentional load (i.e., increasing numbers of tracked targets) under a constant perceptual load (i.e., number of moving stimuli on any given trial; Culham et al., 2001; Jovicich et al., 2001; Tomasi et al., 2006). In their original work, Culham et al. distinguished between task-only brain regions (activation during tracking that is not modulated by tracking load) and load-dependent brain regions (activation during tracking that is modulated by the number of stimuli tracked). Load-dependent alternations of activity are of particular interest because they result from increased attentional demands during a constant perceptual load.

In light of the known response differences of the PIVC and PIC to visual object motion, we predicted that attentional tracking would enhance activity in the PIC and, at the same time, suppress activity in the PIVC. Further, we expected that activity in the PIVC would be more suppressed the greater the attentional load. These hypotheses were examined using functional magnetic resonance imaging (fMRI). Activity during attentional tracking was computed relative to passive viewing of object motion, which served as baseline. For a control experiment, a blank baseline was included and the magnitude of activity change during attentional tracking and passive viewing of object motion was compared.

\section{Materials and Methods}

Participants. Twenty-five right-handed participants (15 females; mean age, $25 \pm 5$ years) from the University of Regensburg student body were recruited for the study. Participants gave informed written consent. The study was approved by the local ethics committee at the University of Regensburg. Each participant performed the attentional tracking task. A subset of participants completed additional scans to localize visual and vestibular ROIs. These scans were performed on $n=12$ participants on a separate day. In addition, $n=8$ participants from the original subject group with independently localized ROIs also participated in a control experiment (see below).

Attentional tracking task. The design of the attentional tracking task, used for the primary experiment, was modeled on previous studies (Culham et al., 2001; Jovicich et al., 2001; Tomasi et al., 2006). Figure $1 a$ shows an example tracking trial. During an initial cueing phase (2s), a subset of disks was highlighted in green. The highlighted disks were the targets to be tracked. Either one, two, three, or four of eight disks in total could serve as targets, creating four levels of attentional load. Nonhighlighted disks served as distractors. All disks had a constant diameter of $0.7^{\circ}$ and were initially arranged in a radial manner, $6^{\circ}$ distant from central fixation. The initial position of each disk was slightly jittered on each trial. During the cueing phase, all disks remained stationary. Thereafter, all disks turned white so that targets were physically indistinguishable from distractors. Then, all disks moved randomly and independently across the screen for $14 \mathrm{~s}$ (speed, $7^{\circ} / \mathrm{s}$; size of tracking field, $27 \times 20^{\circ}$ ). Motion trajectories were constrained to avoid overlap or collisions with other disks or with the central fixation spot. Disks were repelled from the boundaries of the screen. Participants were required to covertly track the target disks while maintaining central fixation. After $14 \mathrm{~s}$, all disks stopped moving and one disk turned blue (response period). Participants were given $2 \mathrm{~s}$ in a two-alternative forced-choice design to decide whether the blue disk was a target or a distractor. In half of the trials of each attentional load condition, the response disk was a target (in the other half it was a distractor). Therefore, chance level was $50 \%$ across all load conditions. After the response period, feedback (a change to green or red in the fixation point for correct or incorrect response, respectively) was provided for $2 \mathrm{~s}$. The total duration of a tracking trial was $20 \mathrm{~s}$. Each tracking trial was followed by a baseline period during which the eight disks moved randomly for $20 \mathrm{~s}$ (using the same constraints for motion trajectories as in the tracking conditions) and participants passively viewed the moving disks while maintaining central fixation. No disks were highlighted for the baseline blocks and participants were explicitly instructed before the experiment not to track any disks during baseline.

Two fMRI runs were performed, with 64 trials each (eight for each tracking condition and 32 baseline trials; run duration, $\sim 21 \mathrm{~min}$ ). Tracking trials were presented in random order. Motion trajectories were computed for each trial a priori and used for all participants.

Before entering the scanner, participants performed a behavioral practice session to familiarize themselves with the tracking task. As a result of this practice, performance in the scanner was, on average, $>80 \%$ correct in each tracking condition (Fig. $1 b$ ). During the practice session, eye tracking was conducted for each participant's right eye using a videobased eye-tracking system (sampling rate, $250 \mathrm{~Hz}$; Cambridge Research Systems). Eye-tracking data from five participants were so noisy that they had to be excluded from analysis. For the remaining participants, the deviation from fixation across trials was low (on average, $\sim 1^{\circ}$ in each tracking condition) and not significantly different between tracking conditions (repeated-measures ANOVA, $F_{(3,19)}=0.50, p=0.69$ ). Eye tracking was not performed during fMRI.

Control experiment: difference between tracking and passive viewing. The attentional tracking task in the control experiment was identical to the task used in the primary experiment, except that a blank baseline was included, during which participants maintained central fixation. During this baseline, the screen was dark and only a central fixation point was presented. There were five conditions in the control experiment (passive viewing of moving disks and attentional tracking, with four different load levels). Each condition was presented eight times, in random order, within each of two fMRI runs and was always followed by blank baseline. Tracking, passive viewing, and baseline were each 20 s long. The duration of each fMRI run was $\sim 27 \mathrm{~min}$. The same motion trajectories as in the primary experiment were used. Due to a replacement in scanner systems, this control experiment was performed in a different scanner environment (see below). Due to constraints of this new environment, the size of the tracking field was limited to $7 \times 5^{\circ}$. Disks had a diameter of $0.15^{\circ}$, moved with a speed of $1.5^{\circ}$, and were initially presented at a distance of $1.3^{\circ}$ from screen center. Despite the decreased screen size, participants still performed well on the tracking task [on average (with SE): track 1, 95\% (2.3); track 2, 91\% (2.0); track 3, 87\% (5.7); track 4, 76\% (3.4)].

Caloric localizer. A subset of participants in the current study $(n=7)$ participated in previous experiments involving caloric stimulation for the localization of area PIVC (three participants in Frank and Greenlee, 2014; four participants in Frank et al., 2016). Five more participants completed the caloric localizer for the current study, using the same 
a

$$
\text { Cueing (2s) }
$$

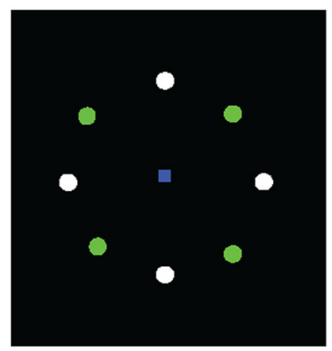

b

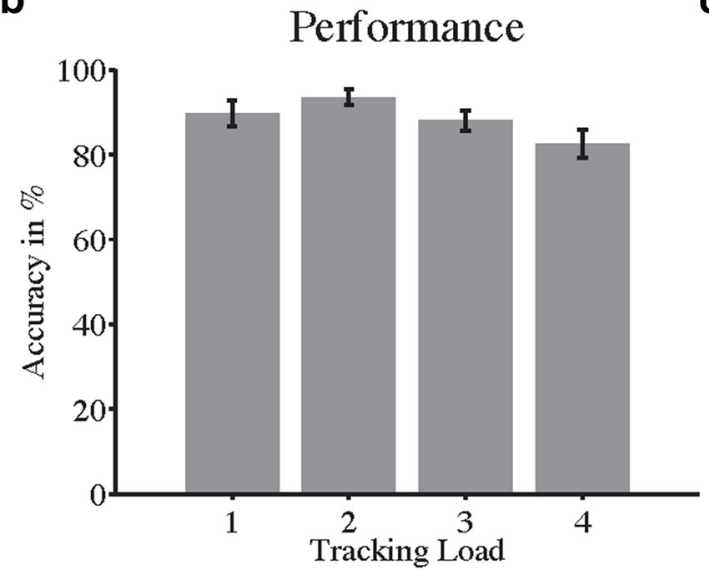

Response (2 s)

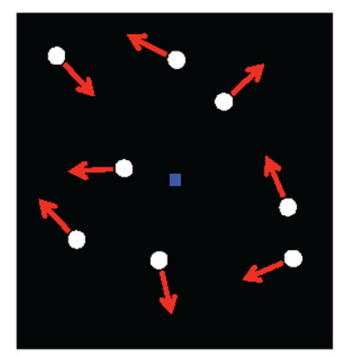

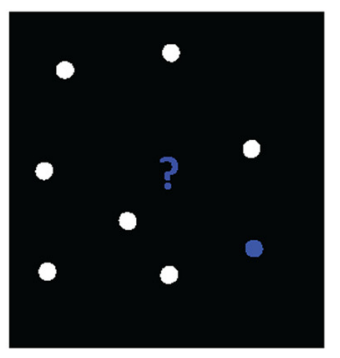

Feedback (2s)

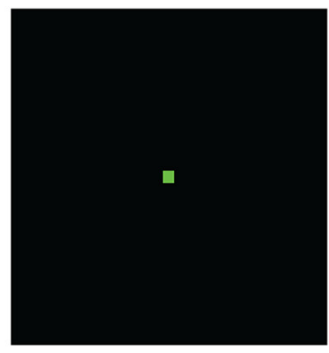

C

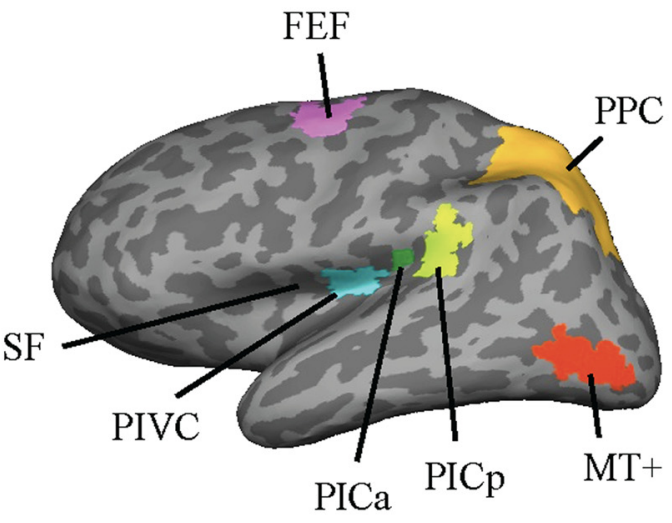

Figure 1. Design of the attentional tracking task. $\boldsymbol{a}$, Each tracking trial started with a cueing phase $(2 \mathrm{~s})$, during which eight disks were arranged radially in the visual periphery and remained stationary. A subset of disks (either 1, 2,3, or 4) was temporarily highlighted in green and served as the tracked targets (in the current example, 4 targets had to be tracked). The more targets that were tracked, the greater was the attentional load. After brief highlighting, the targets turned white, and all disks moved randomly, at a constant speed, across the screen. Disks never overlapped or collided and were repelled from each other and from the borders of the display. Participants were instructed to maintain central fixation and to track the targets covertly, using their attention. After $14 \mathrm{~s}$ of tracking, all disks stopped moving, and one disk was highlighted in blue. Participants were given $2 \mathrm{~s}$ to indicate whether the blue disk was a tracked target or a distractor. At the end of each trial, feedback (green or red fixation for correct or incorrect responses) was provided for $2 \mathrm{~s}$. In the primary experiment, each tracking trial was followed by a baseline trial, during which the disks moved randomly across the screen for $20 \mathrm{~s}$, while participants maintained central fixation. On baseline trials, none of the disks was highlighted and participants passively viewed the moving disks. In the control experiment, each tracking trial was followed by a blank baseline, during which participants maintained central fixation. $\boldsymbol{b}$, Average behavioral performance (with SE) in each of the four tracking conditions of the primary experiment (25 participants; chance level, 50\%; see Materials and Methods for details). c, Locations of ROls, shown on the inflated left hemisphere of a typical participant (dark gray, sulci; light gray, gyri). SF, Sylvian fissure (also called lateral sulcus); PICa/PICp, anterior/posterior clusters of the PIC area; PPC, posterior parietal cortex; MT+ is V5. The PIVC was defined by means of caloric stimulation, whereas the PIC and MT + were defined based on stimulation with visual motion. The FEF was localized by means of activation during saccades. The PPC was defined based on anatomical landmarks (parietal gyrus and sulcus).

parameters as described in Frank et al. (2016). In this localizer scan, caloric temperature stimuli with heat placed in one ear $\left(45^{\circ} \mathrm{C}\right)$ and cold in the other $\left(22^{\circ} \mathrm{C}\right)$ alternated with periods of bilateral neutral stimuli (both ears warm, $36.5^{\circ} \mathrm{C}$ ). In Frank and Greenlee (2014), slightly different temperatures were used (hot, $47.5^{\circ} \mathrm{C}$; cold, $7.5^{\circ} \mathrm{C}$; warm, $31^{\circ} \mathrm{C}$ ). Area PIVC was defined by means of more pronounced activation in caloric stimulation versus neutral (baseline) trials. The location of the PIVC in an example participant is shown in Figure $1 c$.

Visual object motion localizer. A standard visual object motion localizer was used (Frank et al., 2014, 2016). In this localizer, periods of visual object motion ( 200 white dots, moving coherently in one of 12 different translational directions for $1 \mathrm{~s}$ each) were followed by periods of static dots. Dots moved with a speed of $15 \%$, had a limited random lifetime between 167 and $333 \mathrm{~ms}$, and did not spatially overlap. Blocks were $12 \mathrm{~s}$ long and there were 48 blocks in total ( 24 with visual motion). Participants maintained central fixation and performed a speeded dimmingdetection task at the fixation spot. The contrast visual motion $>$ static was used to identify the PIC in the posterior Sylvian fissure. Separate anterior and posterior PIC clusters were observed (Frank et al., 2016) and treated separately in the following analyses. We also used this visual motion localizer to define area MT+ as a control region. ROI locations in an example participant are shown in Figure $1 c$.

Frontal eye field localizer. The frontal eye fields (FEFs) were defined by contrasting activation during periods of saccades with activation during periods of central fixation (Kimmig et al., 2001; Fig. 1c). Participants maintained fixation on a central disk that jumped to different locations on the horizontal screen axis for $12 \mathrm{~s}$ (each location, $1 \mathrm{~s}$ ). Successive locations were not predictable by the participant. Periods of saccades were followed by central fixation for $12 \mathrm{~s}$. In total, there were 48 blocks (24 with saccades).

Posterior parietal cortex. The posterior parietal cortex was defined using the automated parcellation of each participant's high-resolution anatomical scan (Desikan et al., 2006), and consisted of the conjunction between the parietal gyrus and sulcus (Fig. 1c).

Stimulus presentation. Stimuli were generated and presented using Psychtoolbox (Brainard, 1997; Pelli, 1997), running in Matlab (MathWorks). For the primary experiment and functional localizer scans, stimuli were back-projected onto a screen at the back of the scanner bore. Participants viewed the screen with a head coil-mounted mirror (viewing distance, $63 \mathrm{~cm}$ ). For the control experiment, stimuli were projected through a window between control and scanner rooms onto a screen located in front of the patient table. Participants viewed the screen with a head coil-mounted mirror (viewing distance, $390 \mathrm{~cm}$ ).

Scanning parameters. MRI data for the primary experiment were collected using a 3 tesla Allegra head-dedicated scanner (Siemens), using a one-channel head coil. For each participant, a high-resolution anatomical scan was acquired using an MPRAGE sequence [time-to-repeat $(\mathrm{TR})=2.25 \mathrm{~s}$; time-to-echo (TE), $2.6 \mathrm{~ms}$; flip angle (FA), $9^{\circ}$; image 
matrix (IM), $256 \times 256$ voxels; voxel-size, $1 \times 1 \times 1 \mathrm{~mm}$; 256 sagittal slices; no interslice gap). fMRI data were collected using a standard T2*weighted echoplanar imaging $(\mathrm{EPI})$ sequence $(\mathrm{TR}=2 \mathrm{~s} ; \mathrm{TE}=30 \mathrm{~ms} ; \mathrm{FA}$, $90^{\circ}$; IM, $64 \times 64$ voxels; voxel size, $3 \times 3 \times 3 \mathrm{~mm}$; 34 axial slices; interslice gap, $0.5 \mathrm{~mm}$ ). fMRI data for the control experiment and for caloric localizer scans in five participants were collected using a 3 tesla Prisma whole-body scanner (Siemens) with a 20 -channel head coil. Parameters of the EPI sequence used for functional imaging were similar to those of the primary experiment $\left(\mathrm{TR}=2 \mathrm{~s}\right.$; TE $=30 \mathrm{~ms} ; \mathrm{FA}, 90^{\circ}$; $\mathrm{IM}, 64 \times 64$ voxels; voxel size, $3 \times 3 \times 3 \mathrm{~mm}$; 32 axial slices; interslice gap, $0.5 \mathrm{~mm}$ ).

Analysis. MRI data were analyzed using Freesurfer and the FSFAST toolbox (Martinos Center for Biomedical Imaging, Charlestown, MA). Each participant's high-resolution anatomical scan was reconstructed and inflated (Dale et al., 1999; Fischl et al., 1999). fMRI data were motion-corrected, coregistered to the individual reconstructed anatomical brain, smoothed with a three-dimensional Gaussian kernel (fullwidth at half maximum, $5 \mathrm{~mm}$ ), and intensity-normalized.

fMRI data were analyzed with a general linear model (GLM) approach. The blood-oxygenation-level-dependent (BOLD) response was modeled with the SPM hemodynamic response function. For the primary experiment, each GLM had five regressors of interest for four tracking conditions and the baseline condition (i.e., passive viewing of moving disks). Only tracking trials where participants responded correctly were included in these regressors. Separate regressors of no interest were used for incorrect tracking trials, the cueing phase, and the response-plusfeedback phase of each tracking trial. In addition, each GLM contained a linear scanner drift predictor and motion-correction parameters as further regressors of no interest. Parameter estimates for the regressors of interest in each ROI were converted to BOLD percentage signal change by subtracting the baseline signal (passive viewing of moving disks) from the signals in each tracking condition. BOLD results were merged across left and right hemisphere ROIs.

To quantify regions as task-general, the BOLD signal was averaged across tracking conditions in each participant and compared with zero (passive viewing baseline) across participants, using a one-sample $t$ test. For the categorization of an area as load-dependent, BOLD signal change in each tracking condition was submitted to a linear regression analysis in each participant and the slope of the regression line, quantifying linear changes in activity by attentional load, was compared with zero across participants with a one-sample $t$ test. To confirm load-dependent effects on the group level with a larger sample of participants $(n=25$, including participants with ROI localizers), participants' parameter estimate maps for each tracking load were normalized into common space and a linear regression across attentional loads was performed for each voxel in each participant. Regression slopes across participants were compared with zero with one-sample $t$ tests. Results of this analysis were corrected for multiple comparisons $[p<0.05$, false discovery rate (FDR) ] and overlaid on the Freesurfer average brain.

Functional imaging data for the control experiment were analyzed as in the primary experiment, except that an additional regressor of interest was included for the blank baseline. Since the purpose of the control experiment was to compare activation during passive viewing with activation during active attentional tracking, only the central $14 \mathrm{~s}$ of each passive viewing block (beginning $2 \mathrm{~s}$ after block start) were modeled. Therefore, the same amount of data was included in regressors for activetracking and passive-viewing conditions. Activations in passive viewing of disk motion and attentional tracking (average across the four tracking loads) were compared with zero (blank baseline) across participants with one-sample $t$ tests. The magnitudes of activation during tracking and viewing were compared across participants with a paired-sample $t$ test. As in the primary experiment, linear slopes across attentional loads were computed in each ROI and analyzed as described above.

ROIs in functional localizer scans were defined on each participant's inflated left and right hemispheres at an FDR-corrected threshold of $p<$ 0.05. In four participants, the PIC anterior (two participants) or PIC posterior (two participants) could not be defined in one hemisphere and, therefore, only the corresponding PIC cluster in the other hemisphere was analyzed for these subjects.

\section{Results}

Figure 2 shows activations in the attentional tracking task used for the primary experiment. Compared with passive viewing of moving disks (Fig. 2, 0 on $y$-axis), the average activation across all load conditions was significantly suppressed in the PIVC $\left(t_{(11)}=\right.$ $-5.2, p<0.001)$, and significantly enhanced in both the anterior and posterior PIC $\left(t_{(11)}=4.6, p<0.001\right.$ and $t_{(11)}=6.9, p<$ 0.001 ; Fig. $2 a-c)$. Moreover, activity in the PIVC was more strongly suppressed the greater the attentional load. The mean slope of linear regressions across load conditions, quantifying the amount of activity change by attentional load, was significantly different from zero $\left(t_{(11)}=-4.5, p<0.001\right.$; Fig. $\left.2 a\right)$. Activity in the anterior PIC decreased with increasing load $\left(t_{(11)}=-4.3, p=\right.$ 0.001 ), but did not fall below that measured during baseline (activation during passive viewing; Fig. $2 b$ ). Load effects were absent in the posterior PIC $\left(t_{(11)}=-1.5, p=0.17\right.$; Fig. $\left.2 c\right)$.

Figure $2 d-f$ shows activations in the MT,$+ \mathrm{FEF}$, and posterior parietal cortex, which served as control regions. The average activation in each of these areas was significantly increased during tracking compared with passive viewing $\left(\mathrm{MT}+: t_{(11)}=10.7, p<\right.$ 0.001; FEF: $t_{(11)}=15.7, p<0.001$; posterior parietal cortex: $\left.t_{(11)}=10.4, p<0.001\right)$, and in each of these areas, activation increased linearly with attentional load, as indicated by the mean slope of percentage signal change versus load $\left(\mathrm{MT}+: t_{(11)}=6.9\right.$, $p<0.001$; FEF: $t_{(11)}=7.2, p<0.001$; posterior parietal cortex: $\left.t_{(11)}=10.0, p<0.001\right)$.

Results of a whole-brain regression analysis across load conditions are shown in Figure 3 ( $n=25$ participants). The average location of the PIVC, based on caloric stimulation (Frank et al., 2016), is indicated by the crosshairs, overlaid on the Freesurfer average brain. As in the ROI analysis, there were inverse attentional load effects at the group-level location of the PIVC, reflecting an increase in suppression with increasing attentional load (Fig. 2a). Indications for an inverse load effect were also evident at the putative group-level location of the anterior PIC (in this case, reflecting a decrease in activation with increasing attentional load; Fig. 2b). However, caution is warranted because the anterior and posterior PIC tend to vary in location and are difficult to define on the group level (but see Frank et al., 2016). In contrast, there were positive load effects in the MT+ and the frontoparietal attention network (increasing activation with increasing attentional load; Fig. $2 d-f$ ), supporting earlier findings (Culham et al., 2001; Jovicich et al., 2001; Tomasi et al., 2006).

Figure 4 shows activations in the control experiment for passive viewing (tracking load, 0 ) and attentive tracking of moving disks. Compared with blank baseline (corresponding to zero on $y$-axis) there was a weak but significant suppression effect in the PIVC during passive viewing of moving disks $\left(t_{(7)}=-2.4, p=\right.$ 0.047; Fig. 4a). Replicating results of the primary experiment, activity of the PIVC during attentive tracking (average across loads) was significantly suppressed compared with baseline $\left(t_{(7)}=-3.8, p=0.007\right)$. The suppression of activity during tracking was significantly stronger than suppression that occurred during passive viewing $\left(t_{(7)}=-2.7, p=0.03\right)$. As in the primary experiment, activity in the PIVC was more suppressed, the greater the attentional load (linear slopes across tracking loads were significantly different from zero: $\left.t_{(7)}=-5.2, p=0.001\right)$.

In the anterior PIC (Fig. $4 b$ ), activation in passive viewing was not significantly different from baseline $\left(t_{(7)}=0.70, p=0.51\right)$. Activation during tracking (all loads) was also not significantly different from baseline $\left(t_{(7)}=1.7, p=0.14\right)$. However, there were trends for increased activity during tracking, in particular in 
a

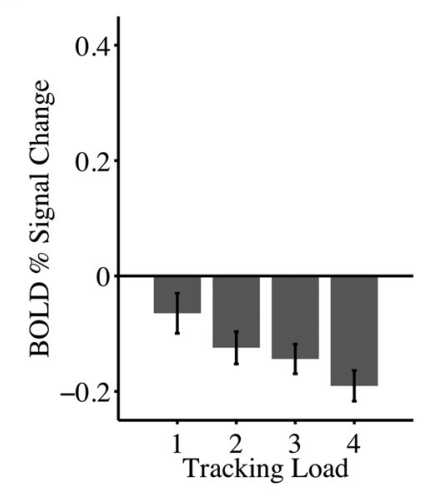

d

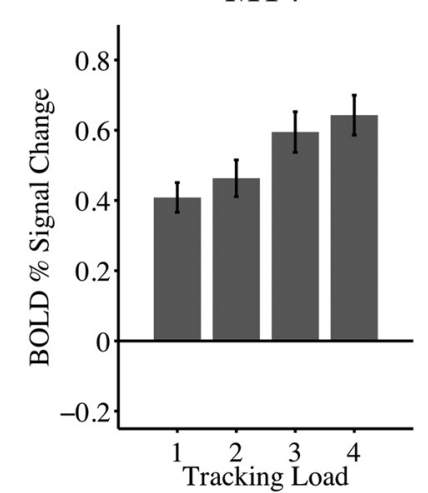

b

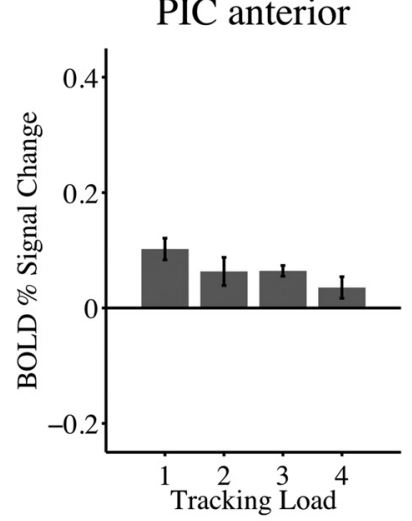

e

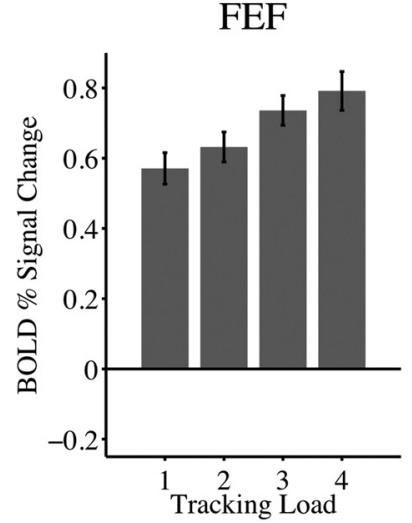

c PIC posterior

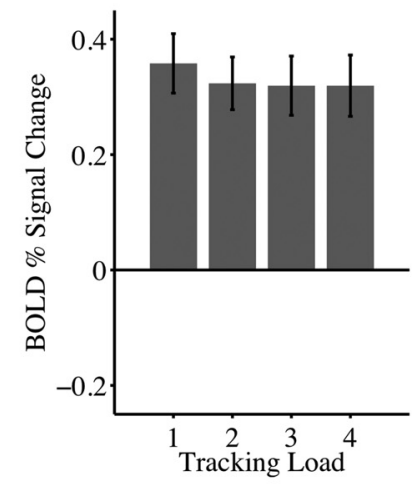

f

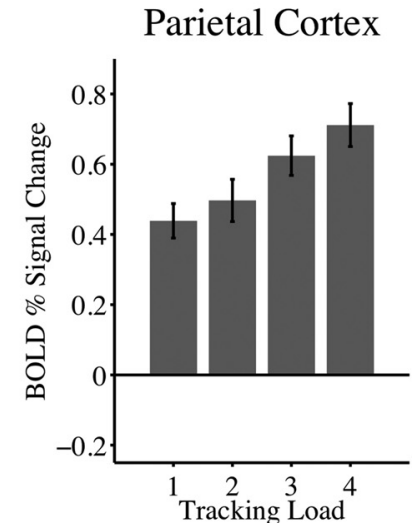

Figure 2. Activations (defined as percentage signal change in the BOLD response) during attentional tracking in the primary experiment. $\boldsymbol{a}-\boldsymbol{c}$, Vestibular areas PIVC, anterior PIC, posterior PIC. $\boldsymbol{d}-\mathbf{f}$, Control regions MT+, FEF, and posterior parietal cortex. Shown are average data (with SE) for each of the four attentional load conditions of the tracking task across 12 participants who completed independent localizer scans for ROI definitions. $y$-axis, BOLD percentage signal change from baseline ( 0 , activation during passive viewing of moving disks). Activity in area PIVC was suppressed during tracking compared with passive viewing, whereas activity in the anterior and posterior PIC was enhanced. The greater the attentional load (i.e., the more targets to be tracked), the more the activity in the PIVC was suppressed, compared with that recorded during baseline trials. Increasing attentional load was associated with declining activity in the anterior PIC, whereas activity in the posterior PIC was similar across load conditions. Each of the control regions (MT+, FEF, posterior parietal cortex) was more strongly activated during tracking compared with passive viewing and activity increased with attentional load.

tracking 1 to 3 disks (Fig. 4b). Activity in tracking and viewing did not differ significantly $\left(t_{(7)}=1.1, p=0.30\right)$. Similar to results of the primary experiment, linear slopes across attentional loads were significantly different from zero $\left(t_{(7)}=-2.6, p=0.03\right)$, indicating that activity in the anterior PIC decreased with increasing attentional demands on the visual task (in particular during the condition where participants tracked four disks; Fig. 4b).

Compared with blank baseline, activity in the posterior PIC was significantly enhanced during passive viewing $\left(t_{(7)}=2.6, p=\right.$ $0.03)$ and during the active tracking of 1-4 moving disks $\left(t_{(7)}=\right.$ $6.8, p<0.001$; Fig. $4 c)$. Activity during active tracking was more pronounced than during passive viewing $\left(t_{(7)}=3.8, p=0.007\right)$. However, linear slopes across attentional loads were not significantly different from zero $\left(t_{(7)}=-0.61, p=0.56\right)$, corresponding to results of the primary experiment.

Activation in each control region (Fig. $4 d-f$ ) was significantly stronger in passive viewing compared with baseline $(\mathrm{MT}+$ : $t_{(7)}=9.3, p<0.001$; FEF: $t_{(7)}=6.2, p<0.001$; posterior parietal cortex: $t_{(7)}=6.7, p<0.001$ ), and in tracking compared with baseline $\left(\mathrm{MT}+: t_{(7)}=12.0, p<0.001\right.$; FEF: $t_{(7)}=12.7, p<0.001$; posterior parietal cortex: $\left.t_{(7)}=11.0, p<0.001\right)$. Moreover, compared with passive viewing, activity in tracking was significantly more pronounced in each ROI $\left(\mathrm{MT}+: t_{(7)}=10.1, p<0.001\right.$; FEF: $t_{(7)}=14.6$, $p<0.001$; posterior parietal cortex: $\left.t_{(7)}=11.3, p<0.001\right)$. As in the primary experiment, activity increased with greater attentional loads (linear slopes across tracking loads were significantly different from zero: $\mathrm{MT}+: t_{(7)}=3.8, p=0.007$; FEF: $t_{(7)}=3.4, p=0.01$; posterior parietal cortex: $\left.t_{(7)}=5.6, p<0.001\right)$.

\section{Discussion}

In this study, we used fMRI to investigate the effect of crossmodal attentional load on activity in the human vestibular cortex. We used a visual tracking paradigm that required participants to covertly track a subset of independently moving targets among moving distractors. To vary the amount of attentional load, the number of tracked targets varied on different trials while the physical stimuli remained identical across different load levels. Two experiments were performed. In the primary experiment, activity during active attentional tracking of moving objects was compared with a passive viewing baseline, during which moving objects were presented while participants did not perform any tracking. In a control experiment, a blank baseline was included and the extent of activity change evoked by passive viewing was compared with that evoked by active attentional tracking.

Results of the primary experiment show that activity in area PIVC was suppressed during attentional tracking compared with passive viewing. In addition, an increase in the number of tracked targets (that is, increasing the attentional load) was associated 


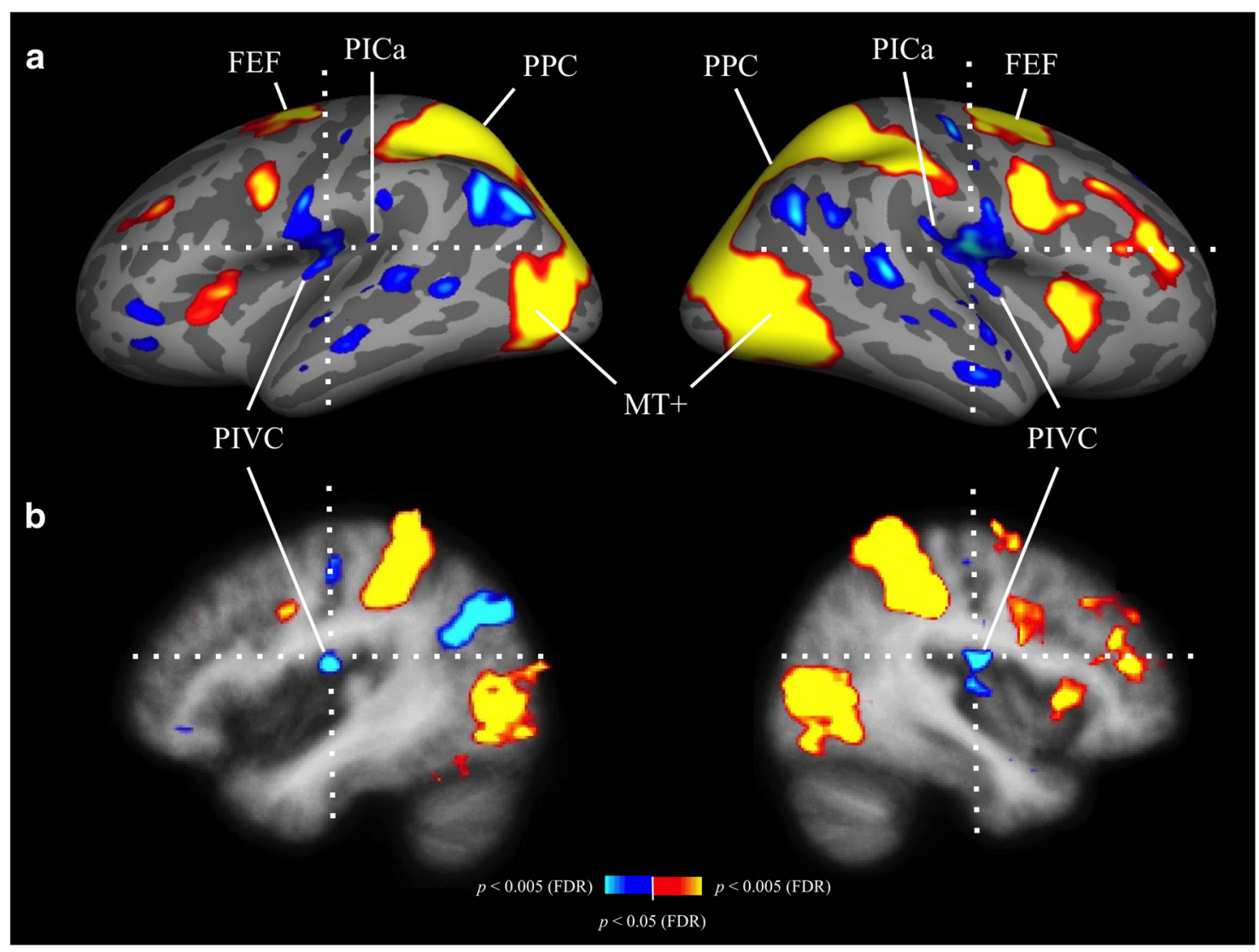

Figure 3. Same as Figure 2, but in a whole-brain analysis, using a larger group of participants $(n=25)$. Linear regressions across tracking loads were performed for each voxel in each participant's brain, and slopes of the regression lines, across participants, were compared with zero, using one-sample $t$ tests. Data shown are corrected for multiple comparisons (FDR, $p<0.05)$. Red-yellow, Regression slopes significantly $>0$ (positive load effect: increasing activation/decreasing suppression with increasing attentional load); blue-white, regression slopes significantly $<0$ (inverse load effect: decreasing activation/increasing suppression with increasing attentional load). (rosshairs are centered on the average position of the PIVC, as determined in an earlier study using caloric stimulation (Frank et al., 2016), at the following average Talairach coordinates: left hemisphere, $x=-43, y=-14, z=17$; right hemisphere, $x=40, y=-14, z=18$. $\boldsymbol{a}$, Results shown on inflated left and right hemispheres of the Freesurfer average brain. An inverse load effect was observed for the PIVC, whereas the MT+ and the frontoparietal attention network showed a positive load effect. The small patch with inverse load effect at the posterior end of the PIVC cluster in both hemispheres appears to be in close proximity to the average location of the anterior PIC, as determined in a recent group analysis (Frank et al., 2016, their Fig. 3b). However, since this cannot be determined with certainty, because the location of anterior and posterior PIC varies substantially between participants, we refer to it as the "putative anterior PIC." $\boldsymbol{b}$, Same as $\boldsymbol{a}$, but in volumetric view.

with stronger suppression of activity in the PIVC. In contrast, activity in the anterior and posterior PIC was enhanced during the attentional tracking task compared with passive viewing. Increasing the attentional demands had no further effect on activity in the posterior PIC, whereas activity in the anterior PIC decreased with increasing attentional load. This decrease of activation in the anterior PIC reflected a decline in excitation, whereas the decline in the PIVC with attentional load appears to reflect increasing inhibition. Activity in control regions (MT+, FEF, posterior parietal cortex) was enhanced during attentional tracking compared with passive viewing and this activity increased with greater attentional loads.

Results of the control experiment show a weak suppression of activity in the PIVC when participants passively viewed the moving objects, compared with a blank baseline, which is in line with previous reports (Brandt et al., 1998). However, the suppression effect was more pronounced when participants directed their visual attention to the moving targets during attentional tracking, suggesting that most of the suppression was driven by attention to the visual stimuli. Overall, our results suggest cross-modal attention effects in area PIVC that are opposite to those obtained in the MT+ and frontoparietal areas.

The results in our control regions replicate previous findings (Culham et al., 1998, 2001; Jovicich et al., 2001; Tomasi et al.,
2006; Howe et al., 2009). Activity in the MT+, FEF, and posterior parietal cortex increased linearly with load during attentional tracking. Therefore, these regions should be categorized as loaddependent, following the nomenclature of Culham et al. (2001).

Our results indicate that the fMRI response pattern differed dramatically between area PIVC and our control regions (MT+, FEF, posterior parietal cortex). Attentional tracking suppressed activity in the PIVC, compared with when participants passively viewed moving objects. This suppression increased linearly as more targets were attentionally tracked. These findings are directly opposite to those on the effects of attentional load in the $\mathrm{MT}+$ and frontoparietal areas. Therefore, activity in the PIVC is suppressed, not enhanced, by load: the greater the attentional load, the greater is suppression of activity in the PIVC. In addition to our ROI analysis, for which we defined the PIVC by means of caloric stimulation, the group analysis of the tracking task in the larger participant sample (Fig. 3) shows an anatomical location of suppressed activity that overlaps well with the average location of the PIVC during caloric stimulation (Frank et al., 2016).

Results of our control experiment suggest that most of the suppression of activity in the PIVC during visual stimulation is caused by attention to visual motion, not due to the passive viewing of these same stimuli. The magnitude of suppression during 
a

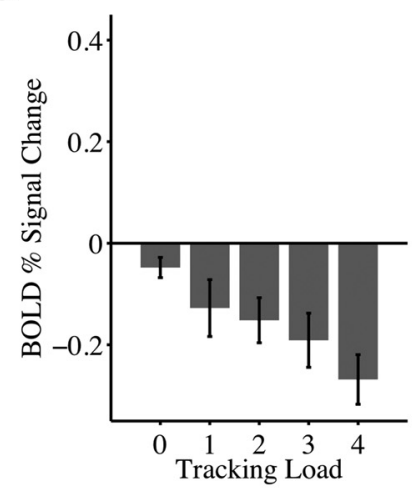

d

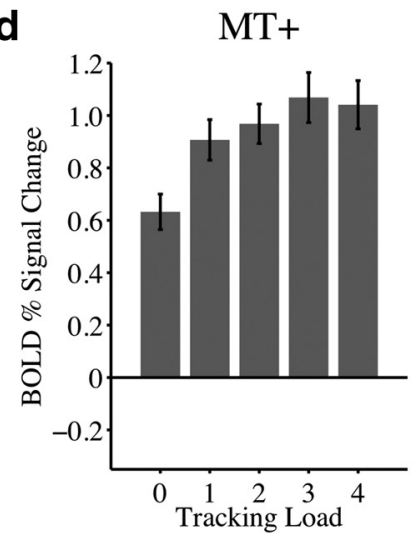

b

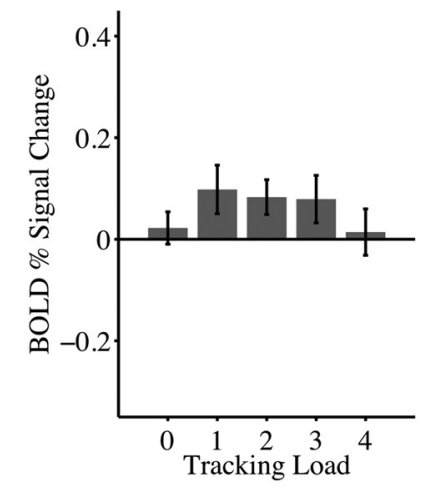

e

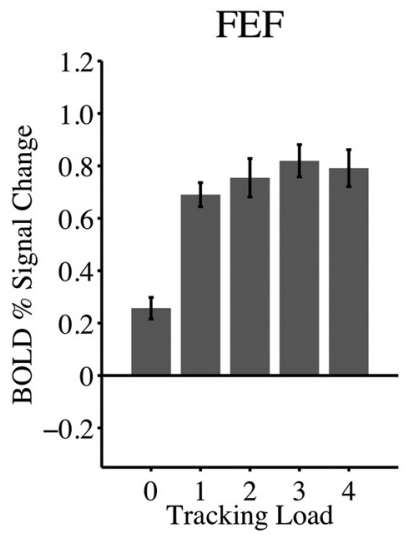

c

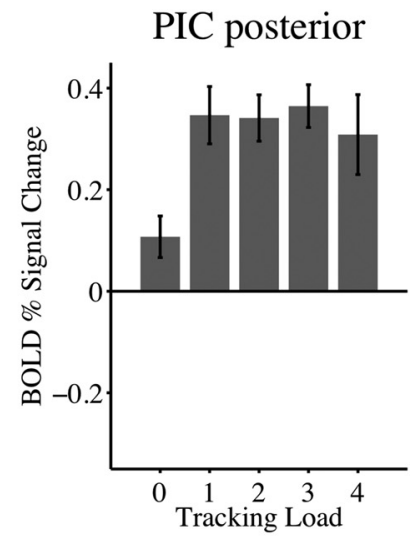

f

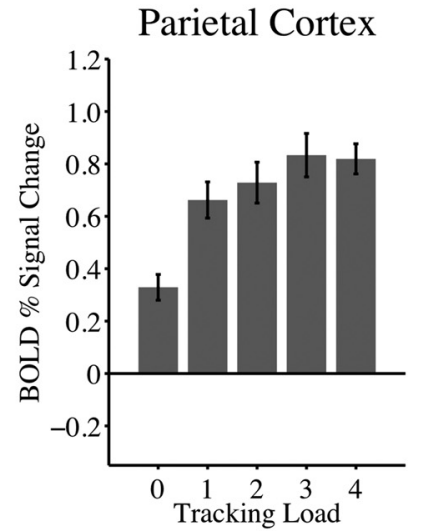

Figure 4. Activations during passive viewing and attentional tracking of moving disks in the control experiment. Here, a blank baseline was used and passive viewing of moving disks was included as another condition of interest, in addition to active multiple-object tracking. Shown are data from eight subjects who also participated in the primary experiment. $x$-axis, Experimental conditions during passive viewing of moving disks ("tracking load 0") and during conditions where 1- 4 disks were tracked. $y$-axis, Average BOLD percentage signal change (with standard error of the mean, SE) from blank baseline ( 0 on $y$-axis). $\boldsymbol{a}$, Compared with blank baseline, activity during passive viewing of moving disks was suppressed in the PIVC, but suppression effects were significantly augmented during increasing levels of attentional load. These inverse load effects were comparable to those found in the primary experiment (Fig. $2 a$ ). $\boldsymbol{b}$, Activity in the anterior PIC was not significantly different from baseline in either passive viewing or tracking and did not differ between these two conditions. However, as in the primary experiment (Fig. $2 b$ ), activity decreased with increasing attentional load (in particular during track 4). c, Conversely, activity in the posterior PIC was enhanced over baseline during both viewing and tracking, and was stronger during tracking than viewing. Replicating previous results (Fig. 2c), the PIC posterior was not modulated by increasing levels of attentional load. $\boldsymbol{d}-\boldsymbol{f}$, Activity in control regions (MT+, FEF, posterior parietal cortex) was significantly enhanced in viewing versus baseline and stronger during tracking than viewing. Activity changes as a function of attentional load were similar to those observed in the primary experiment (Fig. 2).

passive viewing of object motion is comparable to the magnitudes measured in previous reports using fMRI and visual object motion cues (Kleinschmidt et al., 2002; Frank et al., 2016). The weak suppression caused by the visual stimulus in the current study does not suggest a hard-wired interaction mechanism between the visual and vestibular sensory systems. Rather, this interaction appears to be more flexible, depending on the allocation of attentional resources to a particular sensory domain.

Cross-modal suppression of activity in the vestibular cortex during visual stimulation has been interpreted as a means to avoid visual-vestibular conflicts (Brandt et al., 1998; Dieterich and Brandt, 2015) and we speculate that the strong suppression effects, caused by cross-modal attention, reflect an increased effort to inhibit conflicting vestibular signals during the visual tracking task. In this framework, the suppression of activity in the PIVC might inhibit modulatory vestibular input to areas processing visual motion signals related to external objects (e.g., MT+, posterior parietal cortex).

Another finding of our study is that the effects in the PIC were different from those found in the PIVC. Activity in the anterior and posterior parts of the PIC was enhanced during tracking compared with passive viewing (even though these effects were less clear for the anterior PIC in the control experiment). Attentional load had no further modulatory effect on the posterior PIC and this region should therefore be categorized as task-only (using the terminology suggested by Culham et al.), supporting general demands of the tracking task. Therefore, even though the PIC posterior is activated, it does not appear to be as critical to this tracking task as such regions as the MT + or parietal cortex. In the anterior PIC, there was decreasing activity in more demanding tracking conditions, which was replicated in the control experiment. In contrast to activity in the PIVC, activity in the anterior PIC did not fall below that measured during baseline in any of the two experiments.

The decline of excitatory activity in the anterior PIC with increasing attentional load during visual object tracking might hint at the function of the anterior part of the PIC cluster. It is known that the PIC responds to stimuli in both visual and vestibular modalities (Frank et al., 2014, 2016), which is suggestive of multisensory integration between visual and vestibular senses (for results in humans, see Billington and Smith, 2015; for results in nonhuman primates, see Chen et al., 2011), rather than mutual 
inhibition as in the case of the PIVC (Brandt et al., 1998; Dieterich and Brandt, 2015). If the anterior part of the combined PIC cluster is particularly involved in self-motion processing evoked by vestibular or visual cues (Greenlee et al., 2016), visual object motion unrelated to self-motion might be a nonrelevant feature for the anterior PIC, leading to a decline of responses if attention is deployed to this nonrelevant feature. However, we want to emphasize that this is speculative, and more data are needed to support such a functional role of the anterior PIC. The current results suggest that the anterior and posterior PIC might differ not only in anatomical location but also in function, given the different response patterns during attentional tracking (Fig. 2).

Finally, our results contribute to an emerging field of research, referred to as "vestibular cognition" (for review, see Mast et al., 2014; Besnard et al., 2015). An increasing number of results suggests that various cognitive processes are subject to influences by the vestibular system and require vestibular input for normal functioning. These processes range from spatial learning, memory, and navigation (Smith and Zheng, 2013), to more complex spatial cognition, such as mental imagery and mental rotation (Mast et al., 2006), or numerical cognition (Hartmann et al., 2012). Even for sensorimotor processes involved in social cognition (e.g., perspective taking), vestibular input might play an important role (Deroualle and Lopez, 2014). Interestingly, just as vestibular cues influence cognitive processes, so cognitive input likewise influences the vestibular system (Hartmann et al., 2012). In our study, we find a neuronal correlate for the influence of a cognitive process (attentional tracking) on activation in the vestibular cortex.

Future studies might examine whether the suppression of activity in the vestibular cortex during an attentionally demanding visual task has a consequence for the perception of vestibular cues related to self-motion (induced, for instance, by simultaneous caloric stimulation). Moreover, it would be interesting to investigate activity in the vestibular cortex during a visual attention task that is more closely related to the putative function of areas PIVC and PIC (i.e., the sensation of self-motion); this could be, for instance, a heading discrimination task that employs dot patterns with differing proportions of noise dots to vary the attentional load.

\section{Conclusion}

Using a visual multiple-object tracking paradigm we investigated whether the proposed center of the human vestibular cortex is subject to cross-modal attentional modulation as has been demonstrated for other perceptual cortical regions. While the visual stimulus remained unchanged across attentional load conditions, suppression of activity in the PIVC increased linearly with the attentional demands of the visual tracking task. Higher attentional loads were associated with decreasing activation in the anterior part of the PIC, whereas no such effect was observed for the posterior PIC. Overall, our results suggest that visual attention has a cross-modal influence on activity in the human vestibular cortex.

\section{References}

Besnard S, Lopez C, Brandt T, Denise P, Smith PF (2015) Editorial: the vestibular system in cognitive and memory processes in mammalians. Front Integr Neurosci 9:55. CrossRef Medline

Billington J, Smith AT (2015) Neural mechanisms for discounting headroll-induced retinal motion. J Neurosci 35:4851-4856. CrossRef Medline

Brainard DH (1997) The psychophysics toolbox. Spat Vis 10:433-436. CrossRef Medline

Brandt T, Bartenstein P, Janek A, Dieterich M (1998) Reciprocal inhibitory visual-vestibular interaction. Visual motion stimulation deactivates the parieto-insular vestibular cortex. Brain 121:1749-1758. CrossRef Medline

Cavanagh P, Alvarez GA (2005) Tracking multiple targets with multifocal attention. Trends Cogn Sci 9:349-354. CrossRef Medline

Chen A, DeAngelis GC, Angelaki DE (2010) Macaque parieto-insular vestibular cortex: responses to self-motion and optic flow. J Neurosci 30: 3022-3042. CrossRef Medline

Chen A, DeAngelis GC, Angelaki DE (2011) Convergence of vestibular and visual self-motion signals in an area of the posterior sylvian fissure. J Neurosci 31:11617-11627. CrossRef Medline

Culham JC, Brandt SA, Cavanagh P, Kanwisher NG, Dale AM, Tootell RB (1998) Cortical fMRI activation produced by attentive tracking of moving targets. J Neurophysiol 80:2657-2670. Medline

Culham JC, Cavanagh P, Kanwisher NG (2001) Attention response functions: characterizing brain areas using $\mathrm{fMRI}$ activation during parametric variations of attentional load. Neuron 32:737-745. CrossRef Medline

Dale AM, Fischl B, Sereno MI (1999) Cortical surface-based analysis: I. Segmentation and surface reconstruction. Neuroimage 9:179-194. CrossRef Medline

Deroualle D, Lopez C (2014) Toward a vestibular contribution to social cognition. Front Integr Neurosci 8:16. CrossRef Medline

Desikan RS, Ségonne F, Fischl B, Quinn BT, Dickerson BC, Blacker D, Buckner RL, Dale AM, Maguire RP, Hyman BT, Albert MS, Killiany RJ (2006) An automated labeling system for subdividing the human cerebral cortex on MRI scans into gyral based regions of interest. Neuroimage 31:968980. CrossRef Medline

Deutschländer A, Bense S, Stephan T, Schwaiger M, Brandt T, Dieterich M (2002) Sensory system interactions during simultaneous vestibular and visual stimulation in PET. Hum Brain Mapp 16:92-103. CrossRef Medline

Dieterich M, Brandt T (2015) The bilateral central vestibular system: its pathways, functions, and disorders. Ann N Y Acad Sci 1343:10-26. CrossRef Medline

Fischl B, Sereno MI, Dale AM (1999) Cortical surface-based analysis: II: Inflation, flattening, and a surface-based coordinate system. Neuroimage 9:195-207. CrossRef Medline

Frank SM, Greenlee MW (2014) An MRI-compatible caloric stimulation device for the investigation of human vestibular cortex. J Neurosci Methods 235:208-218. CrossRef Medline

Frank SM, Baumann O, Mattingley JB, Greenlee MW (2014) Vestibular and visual responses in human posterior insular cortex. J Neurophysiol 112: 2481-2491. CrossRef Medline

Frank SM, Wirth AM, Greenlee MW (2016) Visual-vestibular processing in the human sylvian fissure. J Neurophysiol 116:263-271. CrossRef Medline

Greenlee MW, Frank SM, Kaliuzhna M, Blanke O, Bremmer F, Churan J, Cuturi LF, MacNeilage PR, Smith AT (2016) Multisensory integration in self-motion perception. Multisens Res 29:525-556. CrossRef

Guldin WO, Grüsser OJ (1998) Is there a vestibular cortex? Trends Neurosci 21:254-259. CrossRef Medline

Hartmann M, Grabherr L, Mast FW (2012) Moving along the mental number line: interactions between whole-body motion and numerical cognition. J Exp Psychol Hum Percept Perform 38:1416-1427. CrossRef Medline

Howe PD, Horowitz TS, Morocz IA, Wolfe J, Livingstone MS (2009) Using fMRI to distinguish components of the multiple object tracking task. J Vis 9(4):10. CrossRef Medline

Jovicich J, Peters RJ, Koch C, Braun J, Chang L, Ernst T (2001) Brain areas specific for attentional load in a motion-tracking task. J Cogn Neurosci 13:1048-1058. CrossRef Medline

Kahane P, Hoffmann D, Minotti L, Berthoz A (2003) Reappraisal of the human vestibular cortex by cortical electrical stimulation study. Ann Neurol 54:615-624. CrossRef Medline

Kimmig H, Greenlee MW, Gondan M, Schira M, Kassubek J, Mergner T (2001) Relationship between saccadic eye movements and cortical activity as measured by fMRI: quantitative and qualitative aspects. Exp Brain Res 141:184-194. CrossRef Medline

Kleinschmidt A, Thilo KV, Büchel C, Gresty MA, Bronstein AM, Frackowiak RS (2002) Neural correlates of visual-motion perception as object- or self-motion. Neuroimage 16:873-882. CrossRef Medline

Lopez C, Blanke O (2011) The thalamocortical vestibular system in animals and humans. Brain Res Rev 67:119-146. CrossRef Medline

Lopez C, Blanke O, Mast FW (2012) The human vestibular cortex revealed 
by coordinate-based activation likelihood estimation meta-analysis. Neuroscience 212:159-179. CrossRef Medline

Mast FW, Merfeld DM, Kosslyn SM (2006) Visual mental imagery during caloric vestibular stimulation. Neuropsychologia 44:101-109. CrossRef Medline

Mast FW, Preuss N, Hartmann M, Grabherr L (2014) Spatial cognition, body representation and affective processes: the role of vestibular information beyond ocular reflexes and control of posture. Front Integr Neurosci 8:44. CrossRef Medline

Mozolic JL, Joyner D, Hugenschmidt CE, Peiffer AM, Kraft RA, Maldjian JA, Laurienti PJ (2008) Cross-modal deactivations during modalityspecific selective attention. BMC Neurol 8:35. CrossRef Medline

Orban GA, Fize D, Peuskens H, Denys K, Nelissen K, Sunaert S, Todd J, Vanduffel W (2003) Similarities and differences in motion processing between the human and macaque brain: evidence from fMRI. Neuropsychologia 41:1757-1768. CrossRef Medline

Pelli DG (1997) The VideoToolbox software for visual psychophysics: transforming numbers into movies. Spat Vis 10:437-442. CrossRef Medline

Pylyshyn ZW, Storm RW (1988) Tracking multiple independent targets: evidence for a parallel tracking mechanism. Spat Vis 3:179-197. CrossRef Medline

Shinder ME, Newlands SD (2014) Sensory convergence in the parietoinsular vestibular cortex. J Neurophysiol 111:2445-2464. CrossRef Medline

Smith PF, Zheng Y (2013) From ear to uncertainty: vestibular contributions to cognitive function. Front Integr Neurosci 7:84. CrossRef Medline

Sunaert S, Van Hecke P, Marchal G, Orban GA (1999) Motion-responsive regions of the human brain. Exp Brain Res 127:355-370. CrossRef Medline

Tomasi D, Ernst T, Caparelli EC, Chang L (2006) Common deactivation patterns during working memory and visual attention tasks: an intrasubject fMRI study at 4tesla. Hum Brain Mapp 27:694-705. CrossRef Medline

zu Eulenburg P, Caspers S, Roski C, Eickhoff SB (2012) Meta-analytical definition and functional connectivity of the human vestibular cortex. Neuroimage 60:162-169. CrossRef Medline 